\title{
Dynamic reconfiguration of human brain functional networks through neurofeedback
}

\author{
Sven Haller ${ }^{\text {a,* }}$, Rotem Kopel ${ }^{\text {b,c }}$, Permi Jhooti ${ }^{\text {g,h }}$, Tanja Haas ${ }^{\text {d }}$, Frank Scharnowski ${ }^{\text {b,c }}$, Karl-Olof Lovblad ${ }^{\text {, }}$ \\ Klaus Scheffler e,f, Dimitri Van De Ville ${ }^{b, c}$ \\ a Service neuro-diagnostique et neuro-interventionnel DISIM, University Hospitals of Geneva, Switzerland \\ ${ }^{\mathrm{b}}$ Department of Radiology and Medical Informatics, University of Geneva, Switzerland \\ c Institute of Bioengineering, Ecole Polytechnique Fédérale de Lausanne, Switzerland \\ d Institute of Radiology, University Hospital Basel, Switzerland \\ e Max Planck Institute for Biological Cybernetic, Tübingen, Germany \\ ${ }^{\mathrm{f}}$ Department of Biomedical Magnetic Resonance, University of Tübingen, Tübingen, Germany \\ ${ }^{g}$ Institute of Research in Art and Design (IDK), Switzerland \\ ${ }^{\text {h }}$ Academy of Art and Design, University of Applied Sciences and Arts Northwestern Switzerland (FHNW), Switzerland
}

\section{A R T I C L E I N F O}

\section{Article history:}

Accepted 5 May 2013

Available online 16 May 2013

\section{Keywords:}

Neurofeedback

Self-regulation

Functional connectivity

Real-time fMRI

\begin{abstract}
A B S T R A C T
Recent fMRI studies demonstrated that functional connectivity is altered following cognitive tasks (e.g., learning) or due to various neurological disorders. We tested whether real-time fMRI-based neurofeedback can be a tool to voluntarily reconfigure brain network interactions. To disentangle learning-related from regulation-related effects, we first trained participants to voluntarily regulate activity in the auditory cortex (training phase) and subsequently asked participants to exert learned voluntary self-regulation in the absence of feedback (transfer phase without learning).

Using independent component analysis (ICA), we found network reconfigurations (increases in functional network connectivity) during the neurofeedback training phase between the auditory target region and (1) the auditory pathway; (2) visual regions related to visual feedback processing; (3) insula related to introspection and self-regulation and (4) working memory and high-level visual attention areas related to cognitive effort. Interestingly, the auditory target region was identified as the hub of the reconfigured functional networks without a-priori assumptions. During the transfer phase, we again found specific functional connectivity reconfiguration between auditory and attention network confirming the specific effect of self-regulation on functional connectivity. Functional connectivity to working memory related networks was no longer altered consistent with the absent demand on working memory.

We demonstrate that neurofeedback learning is mediated by widespread changes in functional connectivity. In contrast, applying learned self-regulation involves more limited and specific network changes in an auditory setup intended as a model for tinnitus. Hence, neurofeedback training might be used to promote recovery from neurological disorders that are linked to abnormal patterns of brain connectivity.
\end{abstract}

(C) 2013 Elsevier Inc. All rights reserved.

\section{Introduction}

Studying how different brain areas interact may hold the key to understand how information is processed in the human brain. Recent developments in data analysis techniques have opened up exciting opportunities to investigate such functional connectivity with functional magnetic resonance imaging (fMRI). The techniques to study large-scale networks using fMRI can be divided into two main approaches. According to the first approach, functional connectivity is measured by

\footnotetext{
* Corresponding author at: Service neuro-diagnostique et neuro-interventionnel DISIM, Hôpitaux Universitaires de Genève, Rue Gabrielle Perret-Gentil 4, 1211 Genève 14, Switzerland. Fax: + 41223727072

E-mail address: sven.haller@hcuge.ch (S. Haller).
}

interregional temporal correlations of the fMRI blood oxygenation level dependent (BOLD) signal (Biswal et al., 1995). This approach requires the choice of a seed region, for which correlation maps can be built. Among other findings, seed-region based approaches lead to the discovery of resting-state functional networks (Fox and Raichle, 2007). The second approach relies on multivariate and data-driven techniques such as independent component analysis (ICA) (Calhoun et al., 2001b; McKeown et al., 1998a, 1998b). ICA can be used to decompose the data into a set of spatial maps and associated time-courses without using pre-defined seed regions (Daubechies et al., 2009). Group-level ICA is a powerful technique to investigate distinct functional networks (Beckmann et al., 2005; Damoiseaux et al., 2006; Greicius et al., 2003).

Many fMRI studies exploring functional connectivity intrinsically assume a static organization. However, recent evidence suggests that 
functional connectivity can be modulated spontaneously (Raichle, 2010), by exogenous stimulation (Buchel et al., 1999), and by learning (Bassett et al., 2011; Lewis et al., 2009). Importantly, changes in functional connectivity have also been linked with the course of a variety of neurological diseases (Fox and Greicius, 2010) as well as the recovery from certain neurological diseases (Wang et al., 2010). Such observations raise the possibility that learning-related changes in functional connectivity can help to accelerate the recovery. This is especially the case if the learning-related changes in functional connectivity can be targeted at the networks involved in the recovery.

A new and promising approach that allows targeting specific regions and networks directly is real-time fMRI (rt-fMRI) neurofeedback (deCharms, 2008; Weiskopf et al., 2004b). The basic principle of rt-fMRI neurofeedback is to present a biofeedback signal extracted online from fMRI BOLD measurements. With the help of such a signal, participants can learn self-regulation of BOLD activity by means of operant conditioning. Several studies have demonstrated the feasibility of self-regulating activity in specific brain areas using rt-fMRI neurofeedback (e.g., deCharms et al., 2004; Posse et al., 2003; Weiskopf et al., 2003, 2004a; Yoo and Jolesz, 2002). Some studies have even shown that self-regulation results in clinical benefits for specific neurological conditions such as chronic pain (deCharms et al., 2005), tinnitus (Haller et al., 2010), and Parkinson's disease (Subramanian et al., 2011). Further, there is preliminary evidence that learning self-regulation of brain activity can lead to changes in functional connectivity (Horovitz et al., 2010; Lee et al., 2011; Rota et al., 2011). However, the studies looking into changes in functional connectivity are limited for two reasons. Firstly, they applied seed-region approaches that limit the investigation of connectivity changes to pre-defined region of interests (ROIs). Secondly, they only investigated connectivity changes during the neurofeedback training phase but they did not look into such changes when participants applied learned self-regulation; i.e., when participants performed previously learned self-regulation without feedback. Especially with respect to clinical applications the transfer condition is more important than the training phase because learned self-regulation along with the accompanying changes in functional connectivity can be voluntarily applied by the patient.

Here we significantly extend the previous investigations of changes in functional connectivity due to neurofeedback by using data-driven techniques that do not require defining a seed region a priori. Because changes in functional connectivity during the neurofeedback training phase might be related to the neurofeedback per se, to learning mechanisms, or both, we included a transfer phase during which participants applied the previously learned strategy in the absence of feedback and hence absence of learning. We hypothesize that our data-driven approach-i.e., independent component analysis (ICA)-can identify changes in functional networks that are related to the neurofeedback target region, in particular, the auditory cortex. Further, we hypothesize that the functional connectivity changes during neurofeedback learning will differ from the changes during applied self-regulation; e.g., only the former will include changes in networks related to feedback processing and reinforcement learning while the latter will demonstrate changes in functional connectivity related to self-regulation.

\section{Materials and methods}

The setup and the experimental procedure were similar to a previously published study (Haller et al., 2010). For readability, the main points are repeated here. For further details, please see Haller et al. (2010). The data used in this study were collected for a previous experiment examining the impact of rt-fMRI on the default-mode network (Van De Ville et al., 2012).

\section{Participants}

Twelve healthy, right-handed individuals (mean age 28.4 years; range 24-33) with normal audition gave written informed consent to participate in the experiment, which was approved by the local ethics committee. Before the experiment, they received written instructions describing that they will learn to regulate their auditory cortex activity with the help of neurofeedback. The instructions included an explanation of the neurofeedback display and recommended as potential regulation strategies to direct attention away from the auditory perception. Further, we explained to the participants that the feedback was delayed by approximately $8 \mathrm{~s}$ (the hemodynamic delay plus the real-time analysis processing time).

\section{fMRI data acquisition}

All experiments were performed on a $3 \mathrm{~T}$ Magnetom Verio whole-body MR scanner, using a standard 12-channel receive head coil (Siemens Healthcare, Erlangen, Germany). Functional data were acquired with a single-shot gradient echo planar imaging sequence (matrix size: $64 \times 64$; isotropic resolution: $3 \times 3 \times 3 \mathrm{~mm}$; echo time TE: $40 \mathrm{~ms}$, repetition time TR: $2000 \mathrm{~ms}$ with 130 repetitions for the auditory localizer runs, 195 repetitions for the training runs and 210 repetitions for the transfer runs). Additionally, we acquired an anatomical T1-weighted structural scan of the whole brain (MPRAGE; $1 \mathrm{~mm}$ isotropic resolution; matrix size $256 \times 256$; 176 sagittal partitions, TE: $3.4 \mathrm{~ms}$, repetition time TR: $2000 \mathrm{~ms}$, TI: $1000 \mathrm{~ms}$ ).

The neurofeedback setup used Turbo BrainVoyager (Brain Innovations, Maastricht, The Netherlands) and custom scripts running on MATLAB (MathWorks Inc., Natick MA, USA). It allowed participants to observe BOLD signal changes in specific brain regions with a delay of less than $2 \mathrm{~s}$ from the acquisition of the image. Head motion was corrected in real-time using Turbo-BrainVoyager.

\section{Experimental procedure}

In the first scanning session, a standard fMRI auditory block-design paradigm was performed to identify each participant's primary auditory cortices. For this, we presented participants with 5 repetitions of $20 \mathrm{~s} \mathrm{bi-}$ lateral auditory stimulation interleaved with $20 \mathrm{~s}$ resting baseline. The auditory stimulus was a sine tone of $1000 \mathrm{~Hz}$ and pulsating at $10 \mathrm{~Hz}$, which is known to induce a strong and long-lasting BOLD response (Haller et al., 2006; Seifritz et al., 2002).

Next, participants took part in 4 rt-fMRI neurofeedback training runs per day repeated over 4 days (with approximately 1 week intervals between training sessions). The training runs were composed of a $30 \mathrm{~s}$ baseline block, followed by 4 repetitions of alternating blocks of $60 \mathrm{~s}$ down-regulation and $30 \mathrm{~s}$ baseline blocks. During the down-regulation blocks, the same pulsating sine tone of $1000 \mathrm{~Hz}$ as in the localizer runs was presented. Participants were presented feedback about their success, which indicated the percentage of signal change compared to the previous baseline block. The visual feedback display was continuously presented during the entire run.

After the neurofeedback training sessions, each participant performed a single self-regulation in the absence of feedback (transfer phase). While changes in connectivity during the training phase might conflate regulation and learning effects, the transfer runs allow assessing the effect of regulation without feedback and thus no further learning-related effects. In the transfer phase, we also included a counting-backwards condition; i.e., the participants were asked to mentally count backwards from 100 in steps of -7 . The purpose of this task was to ascertain a control task with cognitive and working memory load, without the specific application of the previously learned self-regulation strategy. The transfer runs were composed of five $20 \mathrm{~s}$ down-regulation (D) blocks interleaved with five counting (C) backwards blocks and eleven rest $(\mathrm{R})$ blocks of the same duration in a RDRCR... design. The block length during the transfer runs was $20 \mathrm{~s}$ as compared to $60 \mathrm{~s}$ during the training runs. During the training runs, participants were asked to try out different down-regulation strategies in the presence of neurofeedback. Therefore, we opted for regulation 
epochs of $60 \mathrm{~s}$. In contrast, as we expect participants to regulate faster during the transfer runs without feedback and further ability to learn, we opted for shorter regulation epochs of $20 \mathrm{~s}$ in agreement with standard block-design fMRI studies (Amaro and Barker, 2006).

\section{Data preprocessing and GLM analysis}

Preprocessing was performed using the SPM8 software (Wellcome Trust Centre for Neuroimaging, Queen Square, London, UK; http:// www.fil.ion.ucl.ac.uk/). The images were corrected for slice time acquisition differences, spatially realigned to the first scan of each run, normalized into MNI space (Montreal Neurological Institute, resampled voxel size: $2 \times 2 \times 2 \mathrm{~mm}$ ) by using the cubic B-spline interpolation, and smoothed with an isotropic Gaussian kernel with $4 \mathrm{~mm}$ FWHM.

To assess if down-regulation was successfully learned, we specified general linear models (GLMs) with regressors for the experimental conditions (i.e. a boxcar function representing down-regulation and baseline blocks convolved with the canonical hemodynamic response function in SPM8). Data of the training runs were high-pass filtered with a cut-off of $1 / 128 \mathrm{~Hz}$ and serial correlations were modeled by the autoregressive model of order 1 . The group level analysis included the main effect of down regulation in the primary auditory cortex, as well as the linear modulation as a function of training days. Statistical maps for the modulation were obtained using a $t$-test for the corresponding contrast and corrected for multiple comparisons using family-wise error (FWE) at $\mathrm{p}<0.05$.

\section{Independent component analysis}

We used group spatial ICA (Calhoun et al., 2001b) to decompose the training run data into independent components using the GIFT toolbox (http://icatb.sourceforge.net/). We applied ICA to the complete training data by concatenating all runs of all training days and all subjects. ICA then decomposed the data into several temporally-coherent functional networks (independent components, ICs). These maps were extracted for each run along with their associated timecourses. We evaluated the number of ICs and the "quality index" (i.e., so-called IQ measure) of the ICASSO algorithm, which runs ICA multiple times and retains the most reproducible centroids of the ICs. We found that 20 ICs resulted in good reproducibility of all components as indicated by the IQ measure, which warrants a stable and robust decomposition by ICA. We also note that group-level ICA by concatenation of runs and subjects is blind to any ordering.

We scaled the intensities at each voxel to spatial z-scores and then performed a one-sample $t$-test over all runs/sessions/subjects to determine the significant contributions for each IC $(p<0.05$, Bonferroni-corrected). Artifactual non-neurological ICs were identified using manual selection. In particular, three out of the 20 components were excluded from further analyses because they reflected artifacts due to head movement or physiological noise (see Table 1, online Supplementary Table 1). We excluded component IC 7 due to the fact that the majority of voxels are in the venous drainage system, despite some "meaningful" voxels in the parieto-occipital region. We repeated analyses with and without the inclusion of this IC, and there was no significant modification of the results.

\section{Functional network connectivity}

We submitted the associated timecourses of the ICs (for each run) to a connectivity analysis similar to Jafri et al. (2008); i.e., Pearson's correlation coefficients were computed for all pairwise combinations of ICs, leading to a $17 \times 17$ connectivity matrix for each of the 192 runs ( 4 runs per session $\times 4$ sessions $\times 12$ subjects). For every pair, we then performed a linear regression of the session effect per subject, followed by a second level analysis of the slopes. For each connection, a non-zero
Table 1

Overview of the 20 independent components. The functionally-relevant networks were named and classified in agreement with Laird et al. (2011). A detailed description of the activation clusters is available as online supplement.

\begin{tabular}{lll}
\hline IC number & Anatomy/function & $\begin{array}{l}\text { Corresponding IC } \\
\text { (Laird et al., 2011) }\end{array}$ \\
\hline 1 & Auditory network & ICN 16 \\
2 & Artifactual/noise & NA \\
3 & Frontal DMN & ICN 2 \\
4 & Pre-motor & ICN 17 \\
5 & Auditory pathway & No correspondence \\
6 & Basal-ganglia & ICN 3 \\
7 & Artifactual/noise & NA \\
8 & Artifactual/noise & NA \\
9 & High-level visual system and attention & Partly ICN 7 and ICN 10 \\
10 & Insula & Partly ICN 4 \\
11 & DMN & Partly ICN 13 \\
12 & DMN & Partly ICN 13 \\
13 & Peri-hippocampus & No correspondence \\
14 & Working-memory network & No correspondence \\
15 & Primary visual V1 & ICN 12 \\
16 & Primary visual V2 & ICN 11 \\
17 & DMN & Partly ICN 13 \\
18 & DMN & Partly ICN 13 \\
19 & Right parietal & Partly ICN 15 \\
20 & DMN & Partly ICN 13 \\
\hline
\end{tabular}

gradual change over sessions was tested using a one-sample $t$-test of the fitted slopes at $\mathrm{p}<0.05$ (Bonferroni-corrected for all possible connections between the 17 ICs).

\section{Instantaneous connectivity changes during applied self-regulation}

To analyze the transfer runs, we performed "back-reconstruction" (Calhoun et al., 2009) of the ICA networks; i.e., for each time point, all the group-level IC spatial maps were fitted to the measured volumes. This way, we obtained 20 timecourses for each transfer run, which we detrended using cubic polynomial fitting. Next, we extracted the timecourses of the ICs that showed changes in connectivity during the neurofeedback training.

\section{Direct comparison of functional connectivity for different conditions}

For all the time courses of the selected ICs, we concatenated the epochs corresponding to the same condition (i.e., baseline and self-regulation for training runs; baseline, self-regulation, and counting backwards for transfer runs); the hemodynamic lag was accounted for by shifting the time courses by $6 \mathrm{~s}$. Then, for the five functional network connections that showed learning effects, we computed the correlation between the ICs' timecourses for corresponding conditions, which resulted in 24 correlation coefficients for training ( 2 conditions, average correlation over 16 runs, 12 subjects) and 36 correlation coefficients for transfer ( 3 conditions, 12 subjects). We performed a Fisher z-transform followed by a) paired $t$-test to confirm the changes in functional network connectivity between baseline and regulation during training; b) paired $t$-test between change in connectivity baseline-regulation during training versus transfer, and c) paired $t$-tests between the different conditions during transfer. The confidence level of these tests was set to $\mathrm{p}<0.05$ (Bonferroni-corrected for the number of functional network connections).

\section{Results}

\section{Rt-fMRI allows reducing BOLD activity in primary auditory cortex}

Over the course of the four neurofeedback training days, participants learned to reduce activity in the neurofeedback target region (see a previous publication of the same data for a detailed description of the down-regulation over time (Van De Ville et al., 2012)). Please 
note that in the neurofeedback training runs, we actually presented an auditory stimulus during the down-regulation blocks, which was not present during the baseline blocks. Hence, participants are not actually down-regulating spontaneous activity in their auditory cortex, but they are learning to reduce stimulus-induced activity. These results confirm our earlier report that voluntarily reducing auditory cortex activity can be learned with the help of neurofeedback (Haller et al., 2010).

\section{Changes in functional network connectivity due to neurofeedback training}

Using group-level ICA, we identified 17 temporally-coherent functional networks that can be related to neurologically related processes such as visual, auditory, and working memory (Laird et al., 2011); i.e., we excluded 3 artifactual and vascular components out of the 20 ICs (Fig. 1). We next investigated the modifications in functional connectivity between these functional networks. We found a significant gradual change over neurofeedback training sessions in 5 connections ( $p<0.05$, Bonferroni corrected for 17 IC components; inset of Fig. 2). The changes in network connectivity as a function of neurofeedback training are summarized in Fig. 3A. The auditory network was identified as the hub of the training related changes; i.e., the connectivity with the IC corresponding to the auditory network changed for several other ICs. In particular, we observed increased functional connectivity along three different axes: (a) brainstem auditory pathway (IC 5), (b) high-level visual and attention networks (IC 9), and (c) a chain of three networks including an early visual cortex network (IC 15) and insula (IC 10). While typical working memory networks are usually less evidently identified in classic resting-state fMRI studies (Damoiseaux et al., 2006; Laird et al., 2011), IC 14 considerably overlaps with anterior and parietal parts of the working memory network that are consistently identified in task-related activation fMRI studies of working memory, in particular in the context of n-back tasks (Braver et al., 1997; Jansma et al., 2000). Note that the confluent parts of IC 14 partially overlap with the motor network, which is, however, not further discussed in the context of the current investigation. For each of the 5 significant connectivity changes, the average correlation of all sessions as well as the change in correlation over training sessions is provided separately for each participant (Fig. 4A). We also notice that the timecourses of the visual networks (ICs 9 and 15) show deactivation during regulation blocks (Fig. 3A). This phenomenon might be explained by eye saccades during the neurofeedback (Wenzel et al., 1996).

\section{Changes in functional network connectivity due to self-regulation}

Changes in functional connectivity occurring during the training runs of neurofeedback might be related to self-regulation, related to the process of learning, or a combination of both. In order to confirm the specific effect of self-regulation on functional connectivity without the potential confound of learning, we performed the direct comparison of functional connectivity for the different conditions. First, we found that, during the training phase, there are significant changes in baseline versus self-regulation for each connectivity modulated by training, see Fig. 4B. Second, we assessed such changes for the transfer runs when participants applied learned self-regulation in the absence of neurofeedback and consequently the inability to learn. We compared baseline versus regulation, as well as baseline versus counting backwards as a cognitively demanding control condition. For baseline versus regulation, we found a significant change between the auditory network (IC 1) and the high-level visual and attention
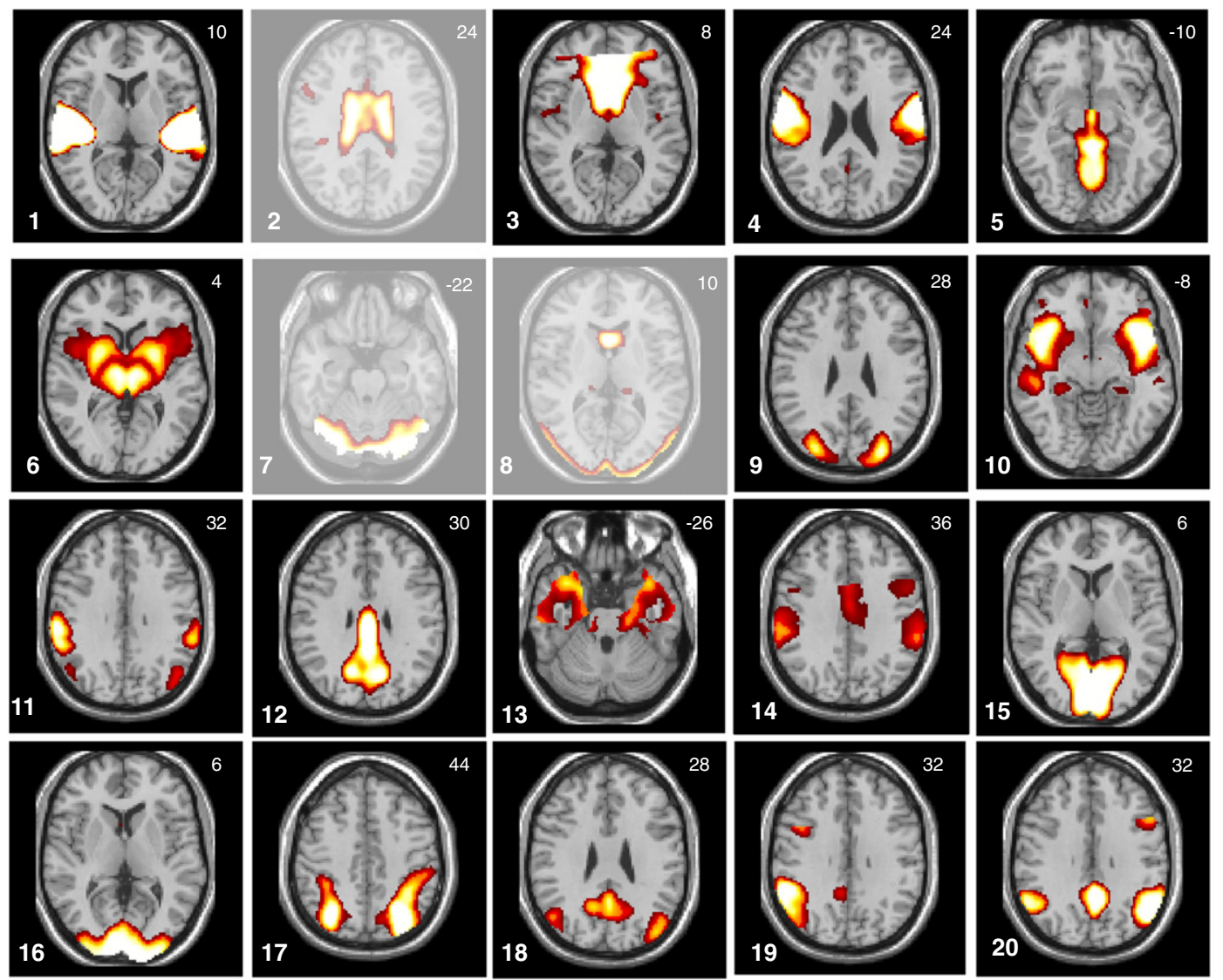

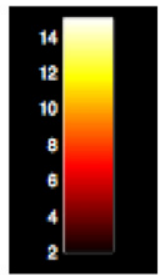

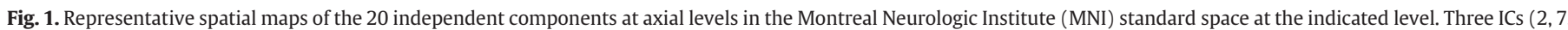

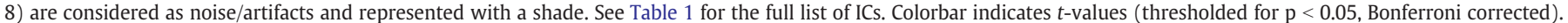




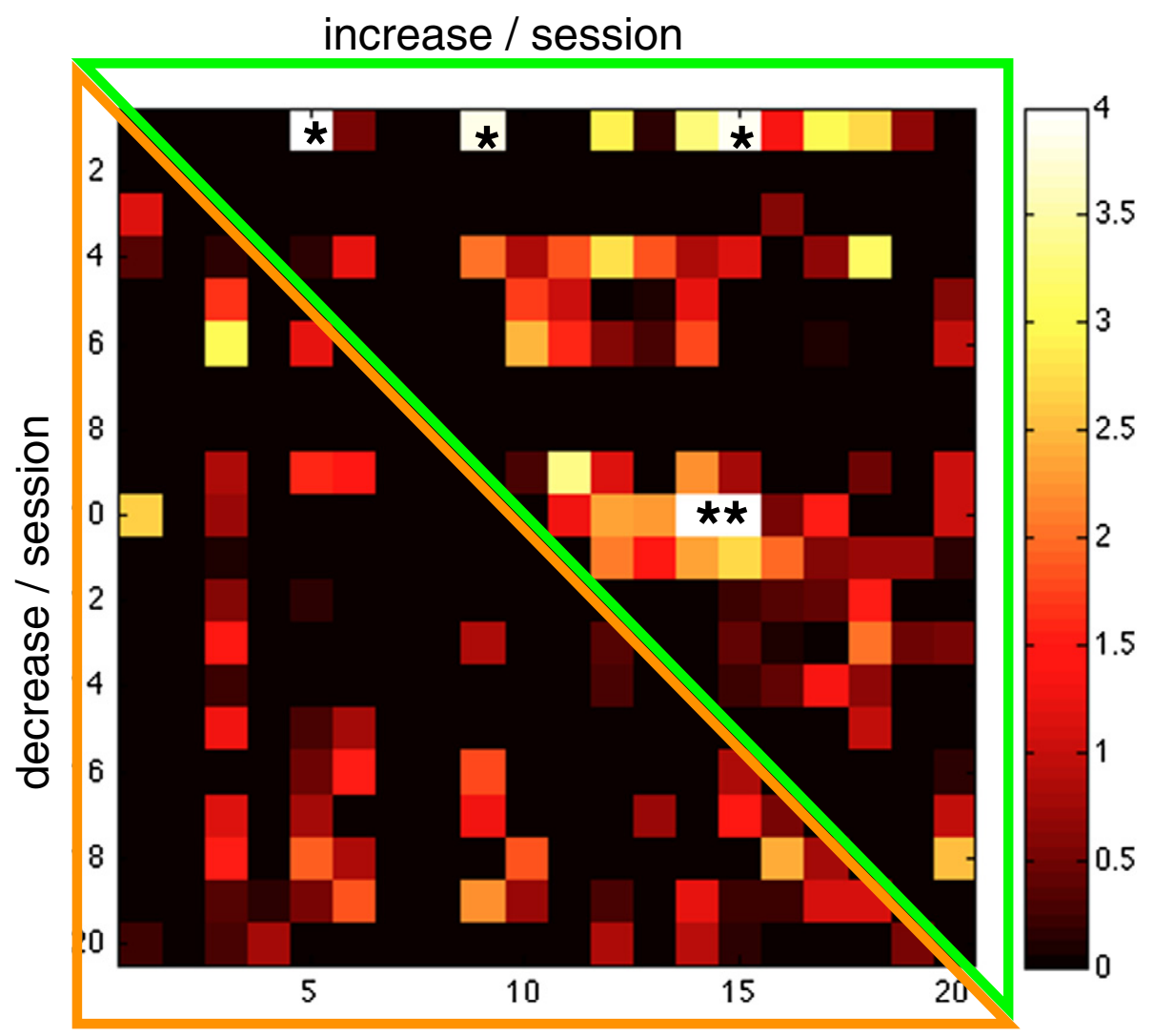

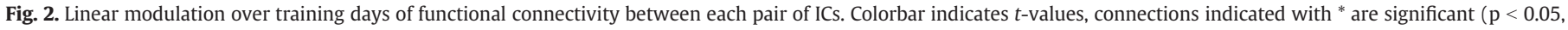
Bonferroni corrected for all possible connections between the 17 selected ICs).

networks (IC 9), see Fig. 4C. Interestingly, connectivity decreased during transfer, while it increased during training. In addition, the connectivity between the auditory network (IC 1) and the auditory pathway (IC 5), as well as with the low level visual (IC 15), working memory (IC 14) and the insular (IC 10) networks did not change significantly during the transfer phase. For the counting backwards condition, we only found one significant change (decrease) for IC 10-IC 15, confirming a specific effect of self-regulation on functional connectivity. While the change in connectivity between insula and low level visual networks during learning might be unexpected at first glance, it is worthwhile mentioning that the analysis of the current investigation is targeted to assess of self-regulation related effects. The analysis of the transfer phase was done using back-reconstruction of ICs defined during the neurofeedback training runs. The analysis is thus biased and not targeted for the specific assessment of counting backwards versus baseline. Interestingly, meta-analysis of task-related fMRI studies includes insula for "counting", "numbers" and basic visual areas for "numbers" (http://neurosynth.org) indicating an involvement of these two regions during the (mental) manipulating of numbers and counting. The counting backwards condition was aimed to confirm the specific effect of self-regulation during the transfer phase and to exclude a global effect on functional connectivity due to the application of a cognitively demanding task, which was indeed the case.

\section{Direct comparison of functional connectivity for different conditions}

We confirmed that all functional network interactions that show learning effects during training (Fig. $3 \mathrm{~A}$ ) have as well a significant change when comparing correlation in baseline versus regulation (Fig. 4B). The differences in connectivity during transfer were significant for IC 1-IC 9 (baseline-regulation) and IC 10-IC 15 (baseline-counting). In addition, we found a significant change in baseline-regulation when comparing training and transfer for two connections; i.e., IC 1-IC 15 and IC 1-IC 9.

\section{Discussion}

We deployed ICA (data-driven exploratory method without a-priori specification of regions of interest) and successfully identified changes in functional network connectivity as a function of neurofeedback training as well as to applying learned self-regulation. Interestingly, the auditory cortex, which was the target area for neurofeedback was identified as the hub of these network changes even though this was not specified a priori. These results show (a) that functional brain networks can change as a function of learning, (b) that rt-fMRI-based neurofeedback training causes network changes that are specific to the neurofeedback target region, and (c) that network changes related to applying learned self-regulation are different from those of the training phase. We will also discuss potential clinical applications of our findings.

\section{Learning related changes of functional connectivity}

Despite the large number of fMRI studies that used functional connectivity measures, only a limited number of them specifically assessed dynamic changes in functional connectivity (Bassett et al., 2011; Lewis et al., 2009). Depending on the complexity of the task, they found differences in complex functional networks when comparing them before and after behavioral training. The neurofeedback training approach that we used significantly advances these earlier findings. Rather than measuring functional-connectivity changes related to a behavioral task, our approach allows to directly target specific brain regions. Also, rather than comparing fMRI scans before and after the behavioral 


\section{A) Training phase with feedback}

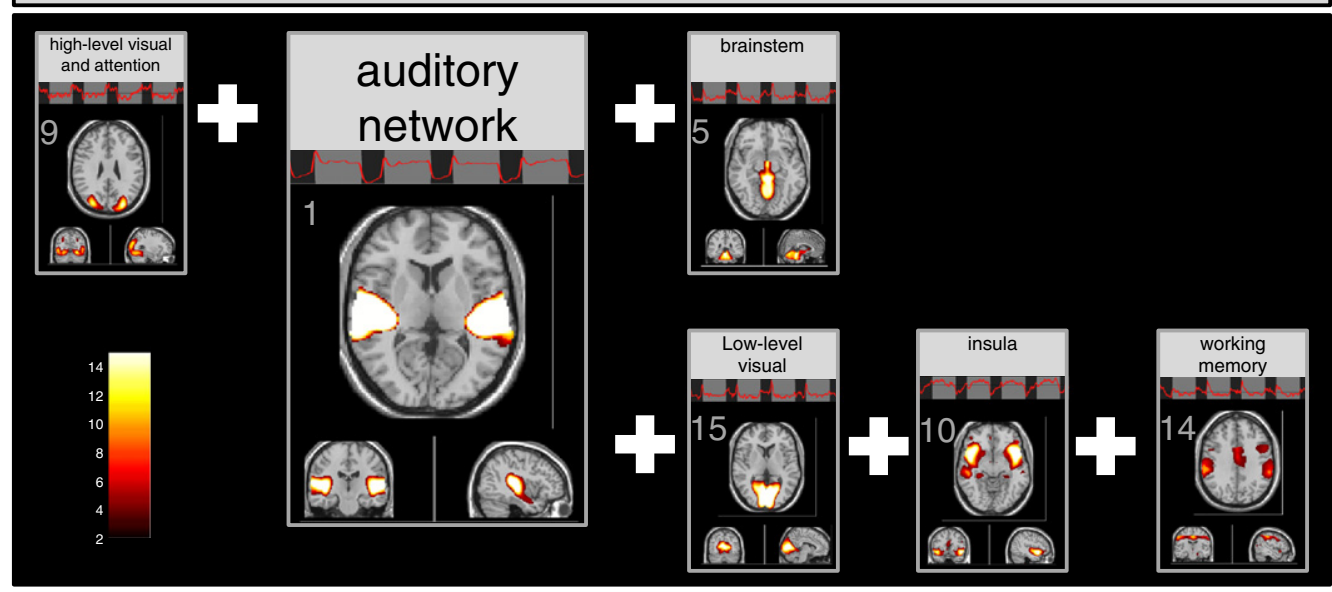

B) Transfer phase without feedback

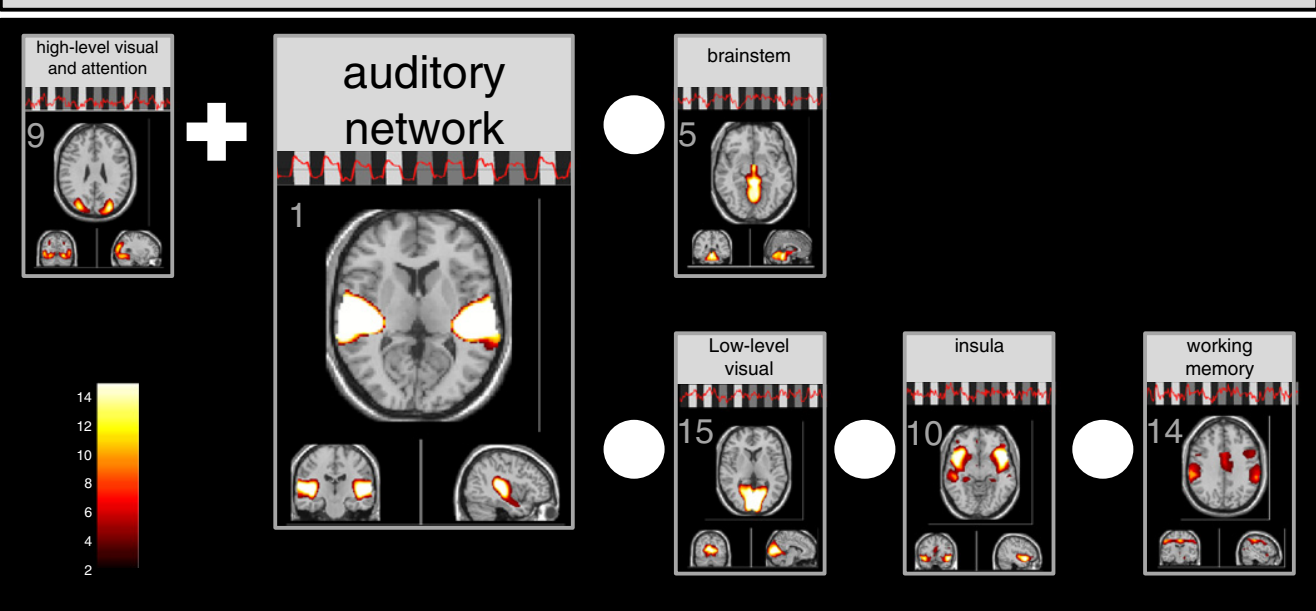

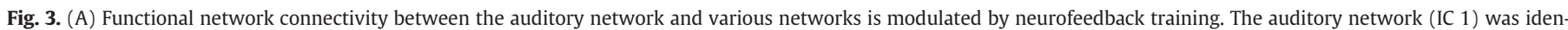

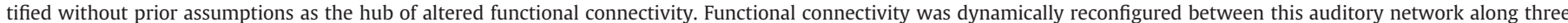

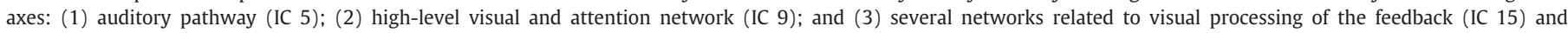

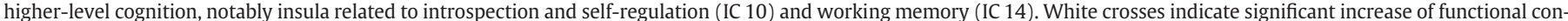

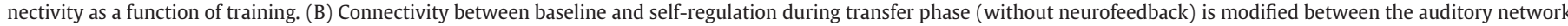

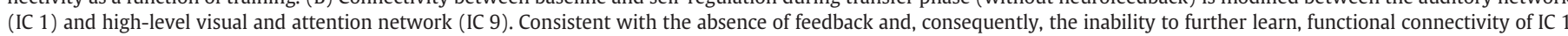
with the visual as well as the memory networks was not modified. Colorbar indicates $t$-values (thresholded for $\mathrm{p}<0.05$, Bonferroni corrected).

training, the neurofeedback learning takes place during scanning. This allows examining progressive learning-related changes in functional connectivity. It also allows distinguishing between learning-related changes and those related to applying learned self-regulation.

Neurofeedback training gradually changes functional connectivity with the auditory target region

The process of learning to self-regulate the auditory target region is mediated by gradual changes in connectivity. Using data-driven analysis, we found that the auditory target region was the hub of these changes (Fig. 3). Note that this network includes bilateral auditory area despite the unilateral ROI in the neurofeedback training, which is consistent with the close anatomic connection of both auditory areas and e.g. bilateral (about $2 / 3$ dominant) contralateral auditory activation to unilateral auditory stimulation (Haller et al., 2006). Our data indicate that the neurofeedback target region does not only change in terms of activity, but in addition alters its connectivity with other networks. During neurofeedback training the functional connectivity of the auditory target region changed along three axes. Firstly, connectivity with the brainstem network (IC 5) increased. This brainstem network (IC 5) includes many pathways, yet in the context of the current investigation the most relevant pathway is the auditory pathway, including the cochlear nuclei, superior olivary complex and inferior colliculi of the tectum and the medial geniculate ganglion of the thalamus. Secondly, connectivity between the auditory target area and higher-level visual and attention networks (IC 9) showed linear increase in connectivity. The self-regulation requires high attentional demands. Moreover, the higher-level visual association area is associated with visual tracking (Laird et al., 2011) required for the tracking of the feedback. Thirdly, connectivity with a widespread network (ICs 15-10-14) including low-level visual as well as insular and working memory areas changed with neurofeedback training. While the early visual network is consistent with the visual feedback, the insular and working memory areas might be attributed to introspective awareness induced by feedback from one's own brain activity and working memory demands when learning to self-regulate. Note that 
A)
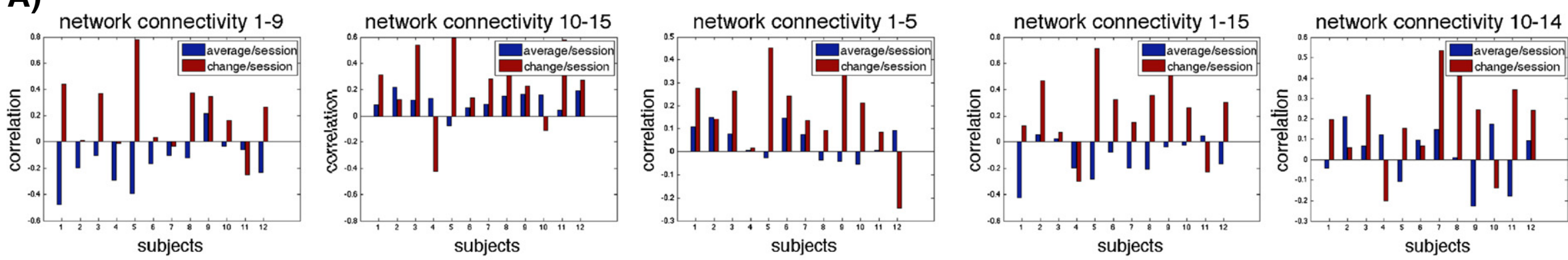

B)
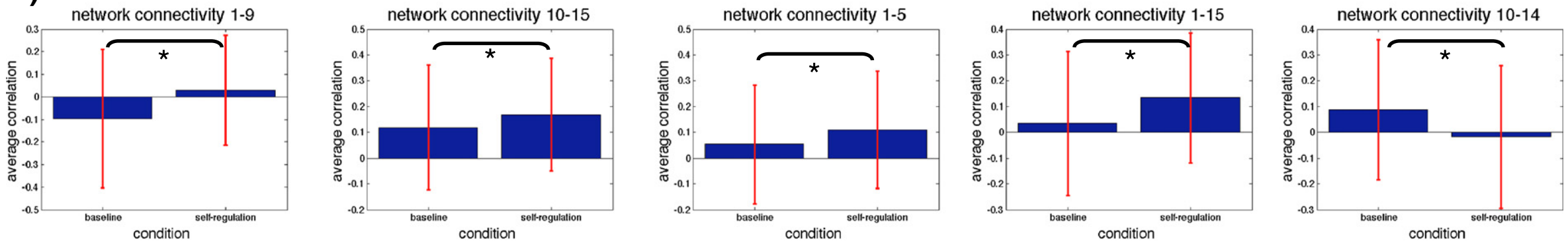

C)
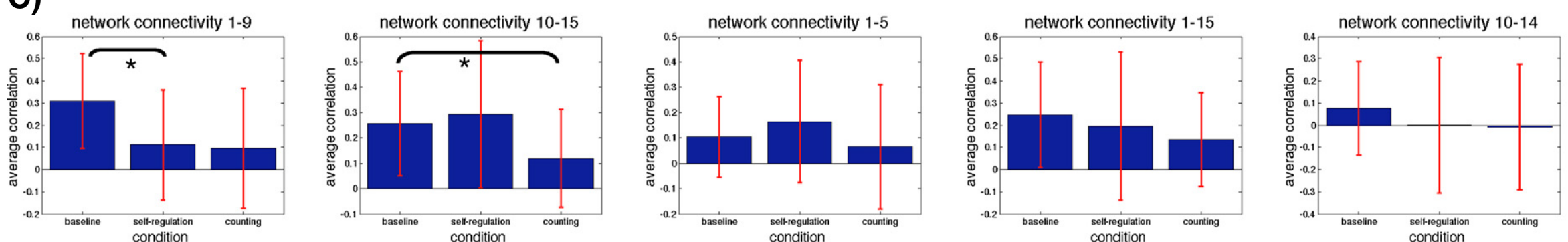

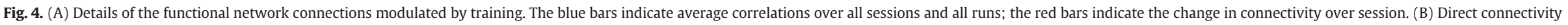

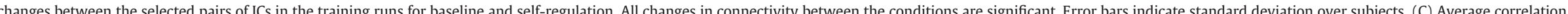

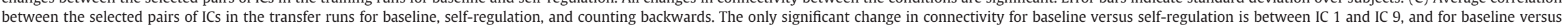
counting-backwards between IC 10 and IC 15. Error bars indicate standard deviation over subjects. 
several previous rt-fMRI neurofeedback investigations found insular activation (for example, see Haller et al., 2010; Subramanian et al., 2011) indicating that this region might be involved in the process of self-regulation per se, while this has not yet been systematically assessed.

While the functional interpretation of each IC that exhibits changes in its connectivity remains somehow speculative, the overall pattern of results suggests that task-relevant functional connections are reinforced. This confirms previous findings of connectivity changes due to neurofeedback learning. For example, Rota et al. (2011) studied linguistic prosody by training participants to self-regulate the right inferior frontal gyrus (rIFG). Using a ROI seed-based functional connectivity analysis, these authors showed that the initially widespread connectivity of the rIFG to frontal and temporal areas decreased over four training sessions. They also showed that the connectivity became more lateralized to the right hemisphere. In another recent study, Horovitz et al. (2010) used similar analysis methods to show that neurofeedback training of the motor area led to increased basal ganglia involvement and bilateral motor cortex connectivity. Finally, Lee et al. (2011) used a multivariate Granger causality analysis to investigate neurofeedback training related changes in the insular cortex. Similar to the studies discussed above, the authors showed that neurofeedback training leads to a reduction of presumably redundant connections and to a strengthening of relevant connections. However, these studies rely on the a priori choice of seed regions and, therefore, do not allow investigating changes in functional connectivity between networks.

\section{Application of previously learned self-regulation causes changes in functional connectivity}

Another limitation of the above-mentioned studies is that they investigated functional connectivity changes only during the neurofeedback training phase. The neurofeedback training, however, is different from applying learned self-regulation after the training. The training involves processing and interpreting the neurofeedback display, testing different strategies, and evaluating the training success. All these components are reflected in the connectivity changes that we found during neurofeedback training (Fig. 3A). Once participants learned self-regulation of the auditory target area, participants can do so even in the absence of neurofeedback. We also confirmed direct changes between baseline and self-regulation for all connections modulated by training (Fig. 4B).

In order to disentangle the confounding effect of learning and to confirm changes in functional connectivity related to self-regulation, participants performed an additional transfer run without feedback and thus without the ability to further learn. In particular, participants were asked to apply the previously learned self-regulation strategy in the absence of feedback. This self-regulation task was contrasted to a cognitively demanding control task (counting backwards). When participants apply their newly acquired self-regulation skill during the transfer run, only the connectivity between the auditory network and attention and high-level visual network changed when comparing baseline against self-regulation, consistent with high demands on attention during self-regulation, which was acquired during the training phase (Figs. 3B and 4C). Learning-related connectivity changes related to introspection, memory demands, or to reinforcement learning were no longer present. Note that the connectivity between the auditory network (IC 1) and the brainstem auditory pathway (IC 5) showed a trend (but non-significant; $\mathrm{p}=0.15$ ) also during self-regulation. In contrast, we observed no effect of the counting backwards condition on this functional connectivity. This confirms that the observed changes in functional connectivity are specific and related to self-regulation learned in rt-fMRI neurofeedback and not simply an effect of performing a cognitively demanding (control) task.

The analysis of the transfer runs was based on back-reconstruction using the 20 ICA maps determined from the training phase data. We mention that alternative approaches of ICA for multiple-condition data have been proposed and could be considered for future work (Beckmann et al., 2006; Calhoun et al., 2001a; Long et al., 2009).

Direct comparison of functional connectivity between training and transfer runs

The direct comparison between training and transfer runs is complicated by the different nature of the training and transfer runs. First, concerning duration, participants were instructed to test several self-regulation strategies during the training runs. In order to have sufficient time to do so, the block length was $60 \mathrm{~s}$. In contrast, during transfer runs, the application of the previously learned self-regulation strategy is faster and thus we opted for a block length of $20 \mathrm{~s}$ in agreement with standard block-design fMRI experiments (Amaro and Barker, 2006). Second, concerning conditions, the training runs had only 2 conditions (baseline and self-regulation). In contrast, the transfer runs had 3 conditions (baseline, self-regulation, and counting backwards) with the latter condition intended as cognitively demanding control condition. Third, concerning repetitions, training runs were repeated 4 times per day in order to give participants sufficient time to learn self-regulation, while the transfer run was performed only once after all learning runs. Consequently, differences in block length and number of runs between training and transfer runs might potentially confound the analysis, and both experimental paradigms are not identical (Fig. 5).

For all functional network connections that showed learning effects during training (Fig. 3A), we confirmed a significant change in correlation when performing a direct comparison between baseline and regulation (Fig. 4B). In addition, the change in connectivity between baseline and regulation was compared between training and transfer. Two connections survived this test. The first one (IC 1-IC 15: auditory-low level visual) confirms our previous result (i.e., significant change in connectivity regulation-baseline within transfer runs). The second one (IC 1-IC 9: auditory-high level visual) seems contradictory because we had before a significant change in connectivity of regulation-baseline both within training and within transfer. However, a closer inspection reveals that the change within training is positive (increase in connectivity), while the change within transfer is negative (decrease in connectivity). In sum, the direct comparison confirms our previous findings and even refines the functional connectivity between auditory and high level visual for regulation applied during transfer.

\section{Limitations}

In this study, all training runs were pooled together for the ICA decomposition, which is used as an unsupervised dimensionality reduction tool prior to further analysis. Similar to PCA, spatial ICA is completely blind to temporal relationships of the data (i.e., temporal permutation does not influence the result), neither is ICA informed about the paradigm. The optimization criterion of ICA (i.e., a surrogate for spatial independence) will be driven by the spatial property 'averaged over time'. However, the fact that post-ICA analysis of the timecourses shows statistically significant changes over time is methodologically valid and does not originate from circularity in the analysis. We previously found learning effects on activity in the target region using a two-level GLM analysis (Van De Ville et al., 2012). Nevertheless, it is worthwhile mentioning that pooling the data might decrease the sensitivity of our data analysis approach. Finally, we mention that the ICASSO algorithm was used to determine the number of components and monitor the robustness of the decomposition.

\section{Outlook and conclusions}

Our approach of combining data-driven analysis tools with a neurofeedback training and transfer phase allowed us to disentangle the connectivity changes when learning to self-regulate and when 


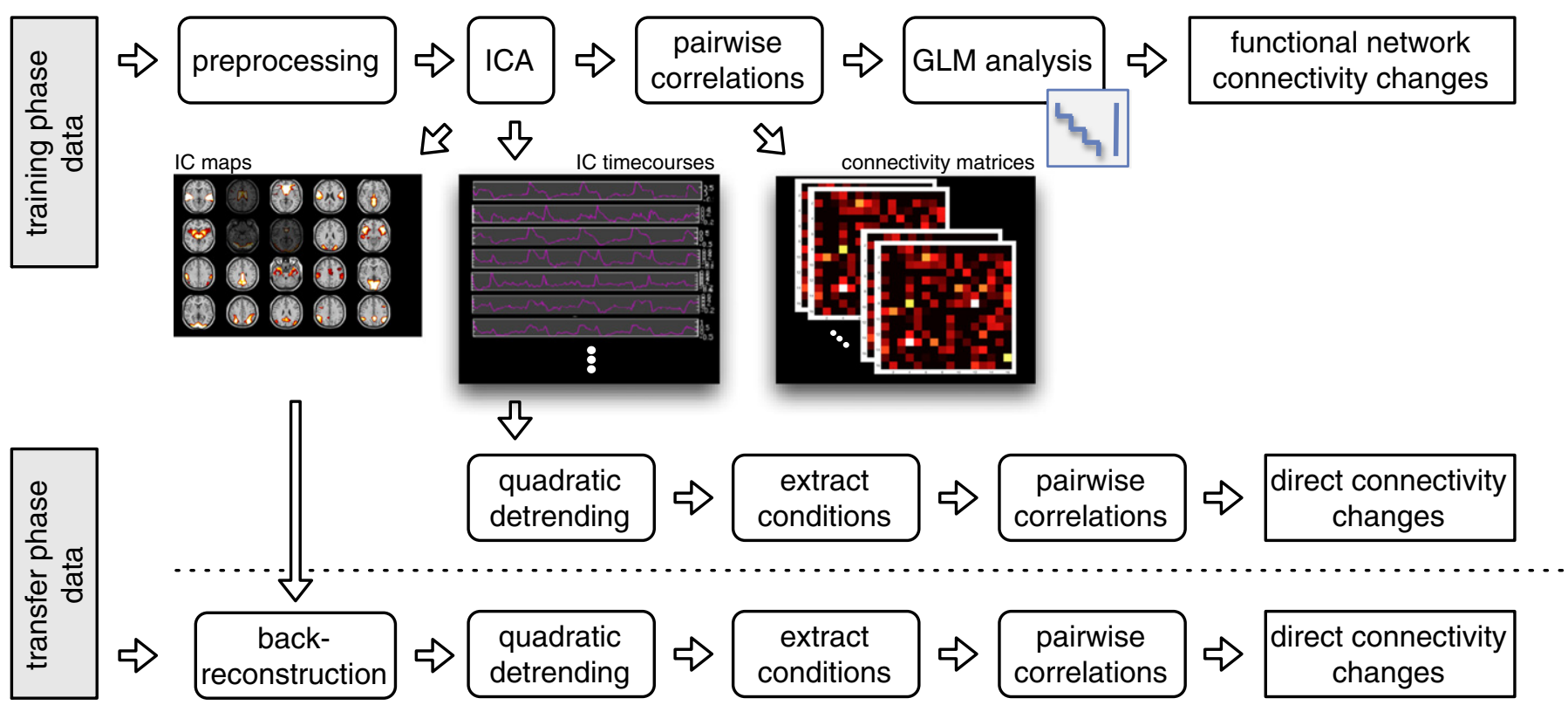

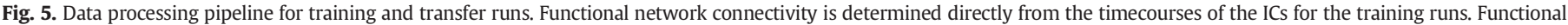
connections that are modulated by training are identified. Direct comparison of connectivity between the conditions is also performed for both training and transfer runs.

applying learned self-regulation. The former is related to widespread changes in learning networks whereas the latter is focused on networks that are specific to the neurofeedback target region.

Importantly, the application of the previously learned self-regulation strategy induces connectivity changes. Hence, the neurofeedback approach can be used to non-invasively and non-pharmacologically manipulate region-specific brain networks. In this sense, neurofeedback might be used to develop strategies to 'normalize' abnormal network activity in patients with certain neurological conditions, such as in tinnitus (Burton et al., 2012; Vanneste et al., 2011) or neglect (Halligan et al., 2003; Husain and Rorden, 2003; Milner and McIntosh, 2005; Vuilleumier et al., 2008). The strategy that was learned by the patient with the help of neurofeedback can be voluntarily applied by the participant and can thus be used concomitant to the conventional therapy. The current setup targeting the auditory region was adapted from the one used for down-regulation of auditory cortex activity in tinnitus patients (Haller et al., 2010). Since we have revealed several changes in network interaction, regulation of brain connectivity might be considered as an explicit target of neurofeedback training for future applications. Previous "classic" ROI-based rt-fMRI neurofeedback studies showed beneficial effects in diseases with clearly defined anatomical target regions (deCharms et al., 2005; Haller et al., 2010; Subramanian et al., 2011). Numerous diseases including Alzheimer disease, depression or psychosis (Broyd et al., 2009; Damoiseaux et al., 2012), however, have clearly defined target regions, but do have documented changes in functional connectivity. Consequently, a functional-connectivity based neurofeedback might complement the ROI based neurofeedback and open access to an increased spectrum of diseases. Moreover, we reason that for example in tinnitus or chronic pain, there is not only an alteration in the primary sensory auditory or sensitive processing, yet also a complex higher-order cognitive alteration related to perception and interpretation of the adverse tinnitus or pain. A connectivity-based approach might better capture such higher-level cognitive components in addition to the lower-level sensory components.

In summary, using data-driven analysis, we found that neurofeedback-based learning induces connectivity changes between the network that encompasses the neurofeedback target region and various other brain networks including those implicated in processing of visual feedback, working-memory and introspection. Subsequently, applying learned self-regulation of brain activity causes instantaneous changes in network interactions that are specific to the neurofeedback target region and attention, while working memory and introspection are no longer required during application of the learned self-regulation strategy in the absence of neurofeedback and are consequently no longer modified. Because learned self-regulation can be voluntarily initiated, it is a promising method to promote recovery from neurological disorders that are linked to abnormal patterns of brain connectivity.

Supplementary data to this article can be found online at http:// dx.doi.org/10.1016/j.neuroimage.2013.05.019.

\section{Acknowledgments}

This work has been supported in part by the Swiss National Science Foundation (under grants 320030_127079/1, PP00P2-123438, PP00P2-146318, PMCDP2-145442, and PZ00P3-131932), and in part by the Center for Biomedical Imaging (CIBM).

\section{Conflict of interest}

No conflicts of interest.

\section{References}

Amaro, E.J., Barker, G.J., 2006. Study design in fMRI: basic principles. Brain Cogn. 60, 220-232.

Bassett, D.S., Wymbs, N.F., Porter, M.A., Mucha, P.J., Carlson, J.M., Grafton, S.T., 2011. Dynamic reconfiguration of human brain networks during learning. Proc. Natl. Acad. Sci. U. S. A. $108,7641-7646$.

Beckmann, C.F., DeLuca, M., Devlin, J.T., Smith, S.M., 2005. Investigations into restingstate connectivity using independent component analysis. Philos. Trans. R. Soc. Lond. B Biol. Sci. 360, 1001-1013.

Beckmann, C.F., Jenkinson, M., Woolrich, M.W., Behrens, T.E., Flitney, D.E., Devlin, J.T., Smith, S.M., 2006. Applying FSL to the FIAC data: model-based and model-free analysis of voice and sentence repetition priming. Hum. Brain Mapp. 27, 380-391.

Biswal, B., Yetkin, F.Z., Haughton, V.M., Hyde, J.S., 1995. Functional connectivity in the motor cortex of resting human brain using echo-planar MRI. Magn. Reson. Med. 34, 537-541.

Braver, T.S., Cohen, J.D., Nystrom, L.E., Jonides, J., Smith, E.E., Noll, D.C., 1997. A parametric study of prefrontal cortex involvement in human working memory. Neurolmage 5 , 49-62.

Broyd, S.J., Demanuele, C., Debener, S., Helps, S.K., James, C.J., Sonuga-Barke, E.J., 2009. Default-mode brain dysfunction in mental disorders: a systematic review. Neurosci. Biobehav. Rev. 33, 279-296.

Buchel, C., Coull, J.T., Friston, K.J., 1999. The predictive value of changes in effective connectivity for human learning. Science 283, 1538-1541. 
Burton, H., Wineland, A., Bhattacharya, M., Nicklaus, J., Garcia, K.S., Piccirillo, J.F., 2012. Altered networks in bothersome tinnitus: a functional connectivity study. BMC Neurosci. 13, 3.

Calhoun, V.D., Adali, T., Pearlson, G.D., Pekar, J.J., 2001. Spatial and temporal independent component analysis of functional MRI data containing a pair of task-related waveforms. Hum. Brain Mapp. 13, 43-53.

Calhoun, V.D., Adali, T., Pearlson, G.D., Pekar, J.J., 2001. A method for making group inferences from functional MRI data using independent component analysis. Hum. Brain Mapp. 14, 140-151.

Calhoun, V.D., Liu, J., Adali, T., 2009. A review of group ICA for fMRI data and ICA for joint inference of imaging, genetic, and ERP data. Neurolmage 45, S163-S172.

Damoiseaux, J.S., Rombouts, S.A., Barkhof, F., Scheltens, P., Stam, C.J., Smith, S.M. Beckmann, C.F., 2006. Consistent resting-state networks across healthy subjects. Proc. Natl. Acad. Sci. U. S. A. 103, 13848-13853.

Damoiseaux, J.S., Prater, K.E., Miller, B.L., Greicius, M.D., 2012. Functional connectivity tracks clinical deterioration in Alzheimer's disease. Neurobiol. Aging 33, 828.e19-828.e30.

Daubechies, I., Roussos, E., Takerkart, S., Benharrosh, M., Golden, C., D'Ardenne, K., Richter, W., Cohen, J.D., Haxby, J., 2009. Independent component analysis for brain fMRI does not select for independence. Proc. Natl. Acad. Sci. U. S. A. 106, 10415-10422.

deCharms, R.C., 2008. Applications of real-time fMRI. Nat. Rev. Neurosci. 9, 720-729.

deCharms, R.C., Christoff, K., Glover, G.H., Pauly, J.M., Whitfield, S., Gabrieli, J.D., 2004 Learned regulation of spatially localized brain activation using real-time fMRI. NeuroImage 21, 436-443.

deCharms, R.C., Maeda, F., Glover, G.H., Ludlow, D., Pauly, J.M., Soneji, D., Gabrieli, J.D., Mackey, S.C., 2005. Control over brain activation and pain learned by using realtime functional MRI. Proc. Natl. Acad. Sci. U. S. A. 102, 18626-18631.

Fox, M.D., Greicius, M., 2010. Clinical applications of resting state functional connectivity. Front. Syst. Neurosci. 4, 19.

Fox, M.D., Raichle, M.E., 2007. Spontaneous fluctuations in brain activity observed with functional magnetic resonance imaging. Nat. Rev. Neurosci. 8, 700-711.

Greicius, M.D., Krasnow, B., Reiss, A.L., Menon, V., 2003. Functional connectivity in the resting brain: a network analysis of the default mode hypothesis. Proc. Natl. Acad. Sci. U. S. A. 100, 253-258.

Haller, S., Wetzel, S.G., Radue, E.W., Bilecen, D., 2006. Mapping continuous neuronal activation without an ON-OFF paradigm: initial results of BOLD ceiling fMRI. Eur. J. Neurosci. 24, 2672-2678.

Haller, S., Birbaumer, N., Veit, R., 2010. Real-time fMRI feedback training may improve chronic tinnitus. Eur. Radiol. 20, 696-703.

Halligan, P.W., Fink, G.R., Marshall, J.C., Vallar, G., 2003. Spatial cognition: evidence from visual neglect. Trends Cogn. Sci. 7, 125-133.

Horovitz, S.G., Berman, B.D., Hallett, M., 2010. Real time BOLD functional MRI neurofeedback affects functional connectivity. Conf. Proc. IEEE Eng. Med. Biol. Soc. 2010, 4270-4273.

Husain, M., Rorden, C., 2003. Non-spatially lateralized mechanisms in hemispatial neglect. Nat. Rev. Neurosci. 4, 26-36.

Jafri, M.J., Pearlson, G.D., Stevens, M., Calhoun, V.D., 2008. A method for functional network connectivity among spatially independent resting-state components in schizophrenia. Neurolmage 39, 1666-1681.

Jansma, J.M., Ramsey, N.F., Coppola, R., Kahn, R.S., 2000. Specific versus nonspecific brain activity in a parametric N-back task. Neurolmage 12, 688-697.

Laird, A.R., Fox, P.M., Eickhoff, S.B., Turner, J.A., Ray, K.L., McKay, D.R., Glahn, D.C., Beckmann, C.F., Smith, S.M., Fox, P.T., 2011. Behavioral interpretations of intrinsic connectivity networks. J. Cogn. Neurosci. 23, 4022-4040.

Lee, S., Ruiz, S., Caria, A., Veit, R., Birbaumer, N., Sitaram, R., 2011. Detection of cerebral reorganization induced by real-time fMRI feedback training of insula activation: a multivariate investigation. Neurorehabil. Neural Repair 25, 259-267.
Lewis, C.M., Baldassarre, A., Committeri, G., Romani, G.L., Corbetta, M., 2009. Learning sculpts the spontaneous activity of the resting human brain. Proc. Natl. Acad. Sci. U. S. A. 106, 17558-17563.

Long, Z., Chen, K., Wu, X., Reiman, E., Peng, D., Yao, L., 2009. Improved application of independent component analysis to functional magnetic resonance imaging study via linear projection techniques. Hum. Brain Mapp. 30, 417-431.

McKeown, M.J., Jung, T.P., Makeig, S., Brown, G., Kindermann, S.S., Lee, T.W., Sejnowski, T.J., 1998. Spatially independent activity patterns in functional MRI data during the Stroop color-naming task. Proc. Natl. Acad. Sci. U. S. A. 95, 803-810.

McKeown, M.J., Makeig, S., Brown, G.G., Jung, T.P., Kindermann, S.S., Bell, A.J., Sejnowski, T.J., 1998. Analysis of fMRI data by blind separation into independent spatial components. Hum. Brain Mapp. 6, 160-188.

Milner, A.D., McIntosh, R.D., 2005. The neurological basis of visual neglect. Curr. Opin. Neurol. 18, 748-753.

Posse, S., Fitzgerald, D., Gao, K., Habel, U., Rosenberg, D., Moore, G.J., Schneider, F., 2003. Real-time fMRI of temporolimbic regions detects amygdala activation during single-trial self-induced sadness. Neurolmage 18, 760-768.

Raichle, M.E., 2010. Two views of brain function. Trends Cogn. Sci. 14, 180-190.

Rota, G., Handjaras, G., Sitaram, R., Birbaumer, N., Dogil, G., 2011. Reorganization of functional and effective connectivity during real-time fMRI-BCI modulation of prosody processing. Brain Lang. 117, 123-132.

Seifritz, E., Esposito, F., Hennel, F., Mustovic, H., Neuhoff, J.G., Bilecen, D., Tedeschi, G., Scheffler, K., Di Salle, F., 2002. Spatiotemporal pattern of neural processing in the human auditory cortex. Science 297, 1706-1708.

Subramanian, L., Hindle, J.V., Johnston, S., Roberts, M.V., Husain, M., Goebel, R., Linden, D., 2011. Real-time functional magnetic resonance imaging neurofeedback for treatment of Parkinson's disease. J. Neurosci. 31, 16309-16317.

Van De Ville, D., Jhooti, P., Haas, T., Kopel, R., Lovblad, K.O., Scheffler, K., Haller, S. 2012. Recovery of the default mode network after demanding neurofeedback training occurs in spatio-temporally segregated subnetworks. Neurolmage 63, 1775-1781.

Vanneste, S., Focquaert, F., Van de Heyning, P., De Ridder, D., 2011. Different resting state brain activity and functional connectivity in patients who respond and not respond to bifrontal tDCS for tinnitus suppression. Exp. Brain Res. 210, 217-227.

Vuilleumier, P., Schwartz, S., Verdon, V., Maravita, A., Hutton, C., Husain, M., Driver, J., 2008. Abnormal attentional modulation of retinotopic cortex in parietal patients with spatial neglect. Curr. Biol. 18, 1525-1529.

Wang, L., Yu, C., Chen, H., Qin, W., He, Y., Fan, F., Zhang, Y., Wang, M., Li, K., Zang, Y., Woodward, T.S., Zhu, C., 2010. Dynamic functional reorganization of the motor execution network after stroke. Brain 133, 1224-1238.

Weiskopf, N., Veit, R., Erb, M., Mathiak, K., Grodd, W., Goebel, R., Birbaumer, N., 2003. Physiological self-regulation of regional brain activity using real-time functional magnetic resonance imaging (fMRI): methodology and exemplary data. Neurolmage 19, 577-586.

Weiskopf, N., Mathiak, K., Bock, S.W., Scharnowski, F., Veit, R., Grodd, W., Goebel, R., Birbaumer, N., 2004. Principles of a brain-computer interface (BCI) based on realtime functional magnetic resonance imaging (fMRI). IEEE Trans. Biomed. Eng. 51, 966-970.

Weiskopf, N., Scharnowski, F., Veit, R., Goebel, R., Birbaumer, N., Mathiak, K., 2004. Selfregulation of local brain activity using real-time functional magnetic resonance imaging (fMRI). J. Physiol. Paris 98, 357-373.

Wenzel, R., Bartenstein, P., Dieterich, M., Danek, A., Weindl, A., Minoshima, S., Ziegler, S., Schwaiger, M., Brandt, T., 1996. Deactivation of human visual cortex during involuntary ocular oscillations. A PET activation study. Brain 119, 101-110.

Yoo, S.S., Jolesz, F.A., 2002. Functional MRI for neurofeedback: feasibility study on a hand motor task. Neuroreport 13, 1377-1381. 